\section{Watching obesity on a new channel}

\section{By C. Simone Fishburn, Senior Editor}

Researchers at the University of California, Irvine, have found a potassium channel Kv1.3 inhibitor that may help treat obesity-related diseases. ${ }^{1}$ The team had previously licensed one inhibitor, ShK-186, to Kineta Inc. for autoimmune indications-the biotech is now planning to test it also in obesity or metabolic syndrome.

Potassium channel Kv1.3 (KCNA3) has long been associated with immune regulation owing to its expression on effector-memory $\mathrm{T}$ cells. ${ }^{2}$ Indeed, four companies have KCNA3 inhibitors in preclinical development for autoimmunity: conoGenetix biosciences $\mathbf{G m b H}$, Axxam S.p.A., Bionomics Ltd. and Circassia Ltd.

However, evidence has been mounting that inhibiting the channel may have additional regulatory functions.

In 2003, a group at Yale University reported the target was involved in regulating body weight. That team produced $\mathrm{KCna}^{-/-}$knockout mice and found the animals were protected from diet-induced obesity and had an increased basal metabolic rate compared with wild-type animals. ${ }^{3}$

In 2011, researchers at Kineta were studying ShK-186 in monkeys and noticed that treated animals consistently had lower cholesterol levels than untreated controls.

These results led Shawn Iadonato, CSO at Kineta, to initiate a collaboration with George Chandy, professor of physiology and biophysics at UC-Irvine and a member of Kineta's scientific advisory board, to test the potential therapeutic use of ShK-186 in obesity.

First the researchers showed that mice receiving ShK-186 gained significantly less weight than vehicle-treated animals when given a high-fat diet to induce obesity.

In obese mice, the peptide decreased adiposity, hyperglycemia and insulin resistance, as measured by normalized blood levels of cholesterol, glucose, hemoglobin A1c (HbA1c), insulin, leptin and thyroid hormones. The molecule also decreased body weight and fatty liver.

Mice fed a normal chow diet showed no weight loss when given ShK-186, suggesting the high-fat diet itself may affect Kcna3-related pathways and make the target more sensitive to inhibition.

Mechanistic studies showed the peptide acts on brown adipose tissue (BAT) and the liver. BAT is a thermogenic form of fat that can take up triglycerides from white adipose tissue (WAT) and use them to generate heat, resulting in increased energy expenditure.

In BAT, the peptide affects $\beta$-oxidation, glycolysis and BAT transcription factors, all of which indicate activation of brown fat metabolism. The compound also increased glucose uptake specifically in BAT and increased sensitivity in an insulin tolerance test, while producing comparable changes in insulin levels to control treatment in a glucose tolerance test.

Together, these results suggest ShK-186 elicits anti-diabetic effects by increasing peripheral insulin sensitivity rather than by increasing insulin secretion from the pancreas.

In the liver, the peptide altered pathways of energy and lipid metabolism, including markers of gluconeogenesis, fatty acids and peroxisome proliferation-activated receptor- $\gamma$ (PPARG; PPAR $\gamma$ )activating metabolites, which most likely contribute to its effects on adiposity and blood markers of obesity.

Finally, ShK-186 decreased levels of tumor necrosis factor- $\alpha$ (TNF- $\alpha$ ) in WAT, suggesting it can decrease obesity-induced inflammation.

Data were reported in Proceedings of the National Academy of Sciences.

\section{Many mechanisms}

Chandy and Iadonato think ShK-186's action on multiple pathways sets it apart from other drugs for obesity-related disorders.

According to Chandy, ShK-186 could work in obesity via a trio of distinct mechanisms: decreased peripheral insulin resistance, decreased obesity-induced inflammation and increased brown-fat activation.

"No other drug acts on type 2 diabetes and cholesterol, and enhances brown-fat activation," Chandy told SciBX.

Kineta completed a Phase I trial of ShK-186 in autoimmune disease in March; the molecule was well tolerated. ShK-186 produced no significant off-target effects in toxicity studies in rats and monkeys.

Next steps include examining ShK-186's role in obesity-related disorders in humans. Kineta has not yet decided whether it will pursue an obesity or metabolic syndrome indication.

Nora Volkow, director of NIH's National Institute on Drug Abuse, thinks the effects ShK-186 has in obesity and diabetes make it well suited to treat patients with metabolic syndrome who are candidates for bariatric surgery.

"What is very impressive is to be able to come out with an intervention that might eliminate the need for surgery," said Volkow, who is interested in the field owing to the role of food addiction in the growing incidence of obesity.

Volkow also believes that BAT activation is a key attribute of the compound. "If you can accelerate energy utilization via peripheral tissues, this is a major issue," she told SciBX.

Kineta has licensed the patent to ShK-186 from Airmid Inc., a company cofounded by Chandy. Kineta is now in discussions with partners to co-develop the compound in metabolic syndrome or obesity.

Fishburn, C.S. SciBX 6(22); doi:10.1038/scibx.2013.537

Published online June 6, 2013

\section{REFERENCES}

1. Upadhyay, S.K. et al. Proc. Natl. Acad. Sci. USA; published online May 27, 2013; doi:10.1073/pnas.1221206110

Contact: K. George Chandy, University of California, Irvine, Calif. e-mail: gchandy@uci.edu 


\section{ANALYSIS}

2. Beeton, C. et al. Mol. Pharmacol. 67, 1369-1381 (2005)

3. Xu, J. et al. Hum. Mol. Genet. 12, 551-559 (2003)

COMPANIES AND INSTITUTIONS MENTIONED

Airmid Inc., Redwood City, Calif.

Axxam S.p.A., Milan, Italy

Bionomics Ltd. (ASX:BNO; OTCBB:BMICY), Thebarton, SA, Australia

\section{TARGETS \& MECHANISMS}

Circassia Ltd., Oxford, U.K.

conoGenetix biosciences GmbH, Martinsried/Planegg, Germany Kineta Inc., Seattle, Wash.

University of California, Irvine, Calif.

Yale University, New Haven, Conn.

National Institutes of Health, Bethesda, Md.

National Institute on Drug Abuse, Bethesda, Md. 